\title{
Study of the molecular clump associated with the high-energy source HESS J1858+020
}

\author{
S. Paron ${ }^{1,2}$, E. Giacani ${ }^{1,2}$, M. Rubio ${ }^{3}$, and G. Dubner ${ }^{1}$ \\ ${ }^{1}$ Instituto de Astronomía y Física del Espacio (CONICET-UBA), CC 67, Suc. 28, 1428 Buenos Aires, Argentina \\ e-mail: sparon@iafe.uba.ar \\ 2 FADU - Universidad de Buenos Aires, Argentina \\ 3 Departamento de Astronomía, Universidad de Chile, Casilla 36-D, Santiago, Chile
}

Received 22 December 2010 / Accepted 20 March 2011

\section{ABSTRACT}

\begin{abstract}
Aims. HESS J1858+020 is a weak $\gamma$-ray source lying near the southern border of the SNR G35.6-0.4. A molecular cloud, composed of two clumps, shows signs of interaction with both the SNR and a nearby extended HII region. In particular, the southernmost clump coincides with the center of the H.E.S.S. source. We study this clump in detail to help us identify the nature of the very-high energy emission.

Methods. We observed the aforementioned molecular clump using the Atacama Submillimeter Telescope Experiment (ASTE) in the ${ }^{12} \mathrm{CO} J=3-2,{ }^{13} \mathrm{CO} J=3-2, \mathrm{HCO}^{+} J=4-3$, and $\mathrm{CS} J=7-6$ lines with an angular resolution of $22{ }^{\prime \prime}$. To complement these observations, we analyzed IR and submillimeter continuum archival data.

Results. From the ${ }^{12} \mathrm{CO}$ and ${ }^{13} \mathrm{CO} J=3-2$ lines and the $1.1 \mathrm{~mm}$ continuum emission, we derive a density of between $10^{3}$ and $10^{4} \mathrm{~cm}^{-3}$ for the clump. We discover a young stellar object (YSO), probably a high mass protostar, embedded in the molecular clump. However, we do not find any evidence of molecular outflows from this YSO that would represent a thermal jet capable of generating the observed $\gamma$-rays. We conclude that the most probable origin of the $\mathrm{TeV} \gamma$-ray emission is a hadronic interaction between the molecular gas and the cosmic rays accelerated by the shock front of the SNR G35.6-0.4.
\end{abstract}

Key words. ISM: clouds - ISM: supernova remnants - gamma rays: ISM - ISM: individual objects: HESS J1858+020

\section{Introduction}

HESS J1858+020 is a weak $\gamma$-ray source that has been detected with the Cherenkov telescope High Energy Stereoscopic System (H.E.S.S.). Though nearly a point-like source, its morphology is slightly extended by $\sim 5^{\prime}$ along its major axis. The source was detected at a significance level of $7 \sigma$ with a differential spectral index of $2.2 \pm 0.1$ (Aharonian et al. 2008). The radio source G35.6-0.4, which was identified as a supernova remnant (SNR) by Green (2009), is seen in projection over the northern border of HESS J1858+020. The author estimated an age of 30000 years for the SNR and a distance of $\sim 10.5 \mathrm{kpc}$. Paron \& Giacani (2010) studied the interstellar medium (ISM) around the very-high energy source and, on the basis of ${ }^{13} \mathrm{CO} J=1-0$ data, identified a molecular cloud composed of two clumps. One of these clumps is seen in projection over the southern border of SNR G35.6-0.4 and displays some kinematical signatures of disturbed gas, while the other clump coincides with the center of HESS J1858+020. On the basis of an IR study, Paron \& Giacani (2010) found evidence of star formation activity in the second aforementioned clump. They suggested that the interaction between the SNR G35.6-0.4 and the molecular gas might be responsible for the $\gamma$-ray emission. They also argued that the star formation processes taking place in the region, could be an alternative or complementary mechanism for explaining the veryhigh energy emission.

In this paper, we present new molecular observations of the dense clump coincident with the HESS J1858+020 center that were carried out to enhance our understanding of the nature of the very-high energy emission.

\section{Observations}

The molecular observations were performed on July 14 and 15, 2010 with the $10 \mathrm{~m}$ Atacama Submillimeter Telescope Experiment (ASTE; Ezawa et al. 2004). We used the CATS345 GHz band receiver, which is a two-single band SIS receiver remotely tunable in the LO frequency range of 324$372 \mathrm{GHz}$. We simultaneously observed ${ }^{12} \mathrm{CO} J=3-2$ at $345.796 \mathrm{GHz}$ and $\mathrm{HCO}^{+} J=4-3$ at $356.734 \mathrm{GHz}$, mapping a region of $90^{\prime \prime} \times 90^{\prime \prime}$ centered at $l=35^{\circ} .577, b=-0.578$ $\left(\mathrm{RA}=18^{\mathrm{h}} 58^{\mathrm{m}} 19.5^{\mathrm{s}}\right.$, Dec $\left.=+02^{\circ} 05^{\prime} 23.9^{\prime \prime}, \mathrm{J} 2000\right)$. We also observed ${ }^{13} \mathrm{CO} J=3-2$ at $330.588 \mathrm{GHz}$ and $\mathrm{CS} J=7-6$ at $342.883 \mathrm{GHz}$ towards the same center mapping a region of $40^{\prime \prime} \times$ $50^{\prime \prime}$. The mapping grid spacing was $10^{\prime \prime}$ and the integration time was $60 \mathrm{sec}$ per pointing in both cases. All the observations were performed in position switching mode. We verified that the off position ( $\left.l=35^{\circ} .478, b=-0.540\right)$ was free of emission.

We used the XF digital spectrometer with a bandwidth and spectral resolution set to $128 \mathrm{MHz}$ and $125 \mathrm{kHz}$, respectively. The velocity resolution was $0.11 \mathrm{~km} \mathrm{~s}^{-1}$ and the half-power beamwidth (HPBW) was $22^{\prime \prime}$ at $345 \mathrm{GHz}$. The system temperature varied from $T_{\text {sys }}=150$ to $200 \mathrm{~K}$. The main beam efficiency was $\eta_{\mathrm{mb}} \sim 0.65$. The spectra were Hanning smoothed to improve the signal-to-noise ratio and only linear or/and some third order polynomia were used for baseline fitting. The data were reduced with NEWSTAR and the spectra processed using the XSpec software package.

To complement the new molecular data, we used the mosaicked images from GLIMPSE and MIPSGAL surveys from the Spitzer-IRAC $(3.6,4.5,5.8$, and $8 \mu \mathrm{m})$ and Spitzer-MIPS 

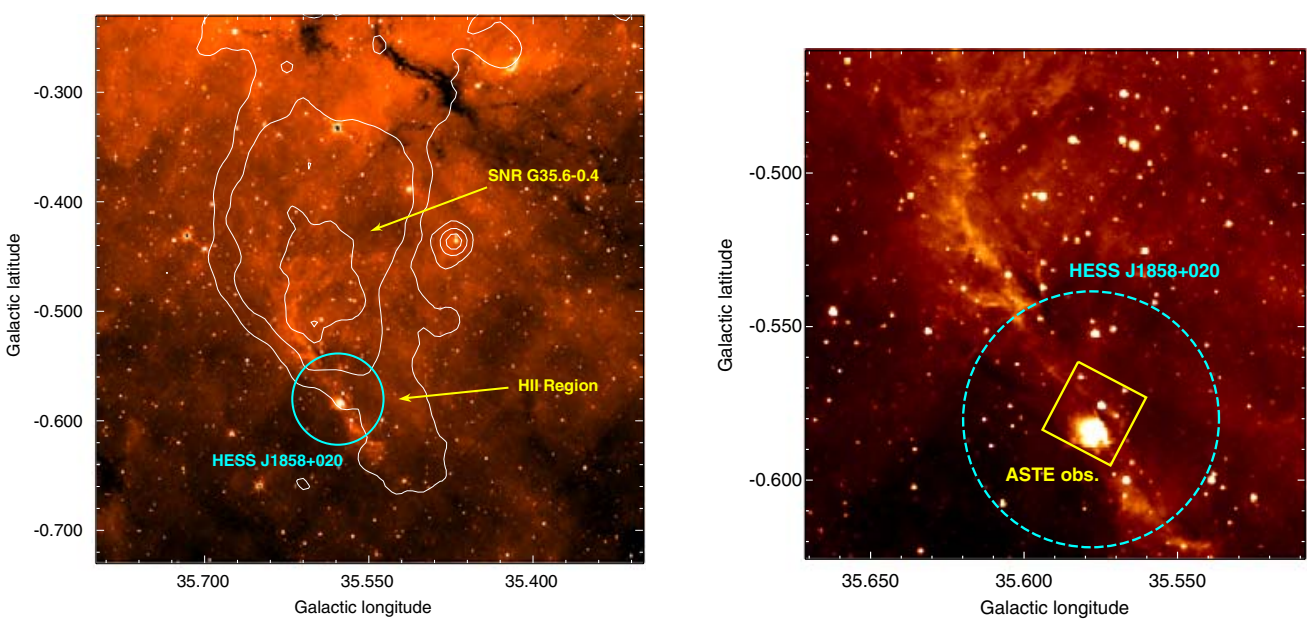

Fig. 1. Left: region of about $30^{\prime} \times 30^{\prime}$ towards SNR G35.6-0.4 presenting the emission at $8 \mu \mathrm{m}$ with contours of the radio continuum emission at $20 \mathrm{~cm}$. The contours levels are 17,22 , and $30 \mathrm{~K}$. The first contour is slightly above the data $3 \sigma_{\mathrm{rms}}$. The circle shows the position and the extension of HESS J1858+020. We note that the SNR is possibly partially superimposed on an HII region. Right: smaller portion of the region displaying the $8 \mu \mathrm{m}$ emission and showing the area mapped with the molecular observations (yellow box).

(24 and $70 \mu \mathrm{m}$ ), respectively. IRAC has an angular resolution of between $1^{\prime \prime} .5$ and $1^{\prime \prime} .9$ and MIPS $6^{\prime \prime}$ at $24 \mu \mathrm{m}$. In addition, we analyzed the continuum emission at $1.1 \mathrm{~mm}$ obtained from the Bolocam Galactic Plane Survey (BGPS), which has a FWHM effective resolution of $30^{\prime \prime}$.

\section{The studied region}

In Fig. 1 (left), we present a region of about $30^{\prime} \times 30^{\prime}$ towards the SNR G35.6-0.4. The image displays the $8 \mu \mathrm{m}$ emission from Spitzer-IRAC with contours of the radio continuum emission at $20 \mathrm{~cm}$. The circle shows the position and the extension of $\sim 5^{\prime}$ of the source HESS J1858+020 (Aharonian et al. 2008). On the basis of the $8 \mu \mathrm{m}$ emission tracing polycyclic aromatic hydrocarbons (PAHs), that partially borders the radio continuum emission extending to the south, we suggest that the SNR G35.6-0.4 partially overlaps an extended HII region, which is likely part of the same complex. This probably explains the confusion about the nature of G35.6-0.4 in the past years (see Green 2009, and references therein). Towards the center of HESS J1858+020, there is an emission peak of $8 \mu \mathrm{m}$, which, as studied by Paron \& Giacani (2010), coincides with a molecular clump detected in the ${ }^{13} \mathrm{CO} J=1-0$ line. Paron \& Giacani (2010) detected evidence of star forming activity that coincides with this clump. This region is catalogued in the IRAS Catalogue of Point Sources (Version 2.0; Helou \& Walker 1988) as IRAS 18558+0201. Figure 1 (right) shows an enlargement of the area of interest indicated by a yellow box the region where the new molecular observations were carried out.

\section{Results and discussion}

Figure 2 (up) shows the ${ }^{12} \mathrm{CO} J=3-2$ spectra obtained towards the observed region. Across the whole area, the main component at $\sim 53 \mathrm{~km} \mathrm{~s}^{-1}$, already detected in the ${ }^{13} \mathrm{CO} J=1-0$ clump studied by Paron \& Giacani (2010), is present. A second, less intense, component is observed mainly towards positive RA and negative Dec offsets (bottom left in the image) with a velocity of $\sim 64 \mathrm{~km} \mathrm{~s}^{-1}$. Owing to a lack of observing time, three positions were not observed (top right of the image). Figure 2 (bottom) displays the ${ }^{13} \mathrm{CO} J=3-2$ spectra observed towards the
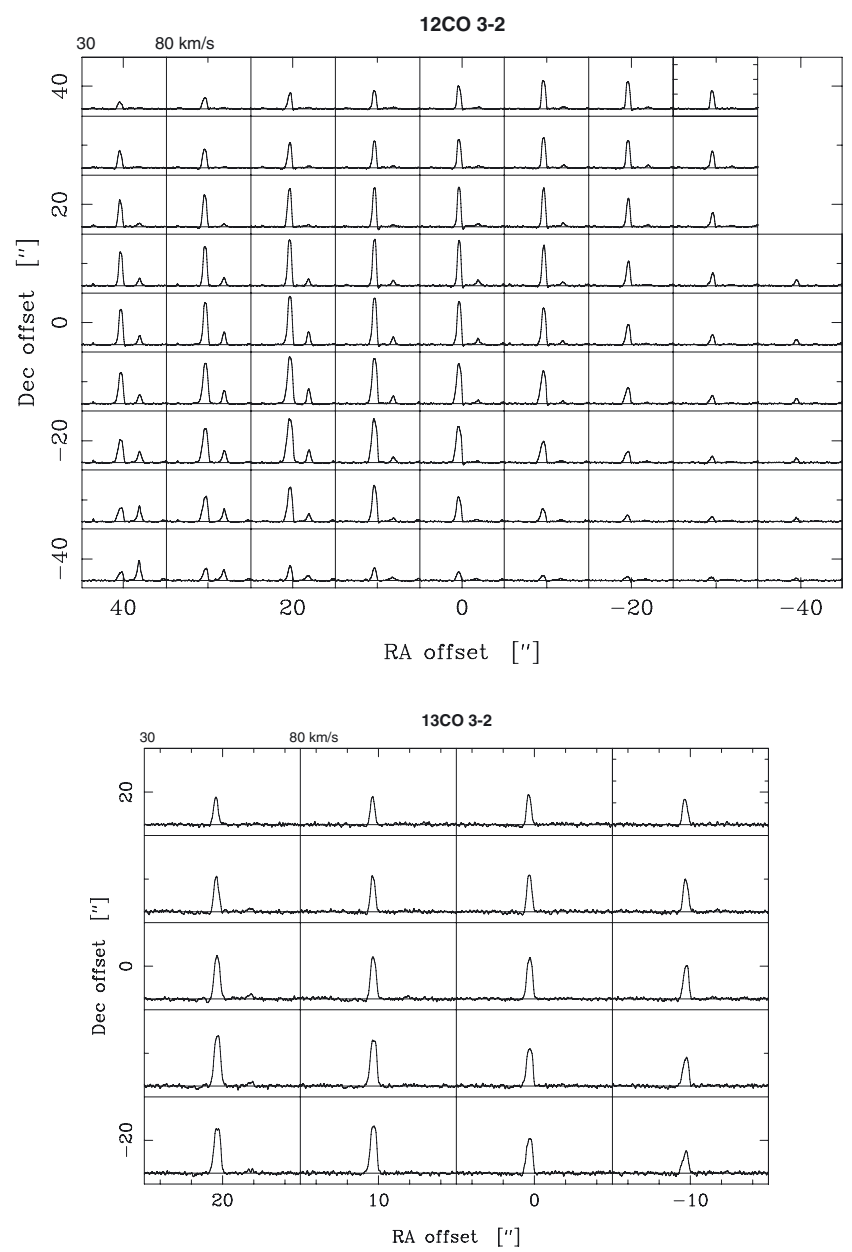

Fig. 2. $U p:{ }^{12} \mathrm{CO} J=3-2$ spectra. Bottom: ${ }^{13} \mathrm{CO} J=3-2$ spectra. The horizontal axis of each spectra is velocity, and ranges from 30 to $80 \mathrm{~km} \mathrm{~s}^{-1}$, while the vertical axis is brightness temperature and goes from -1 to $7 \mathrm{~K}$. The center, i.e. the $(0,0)$ offset, in both lines is the same.

central $\sim 20$ square arcseconds. In the observed area, this line has a unique component centered at $\sim 53 \mathrm{~km} \mathrm{~s}^{-1}$. In both cases, 


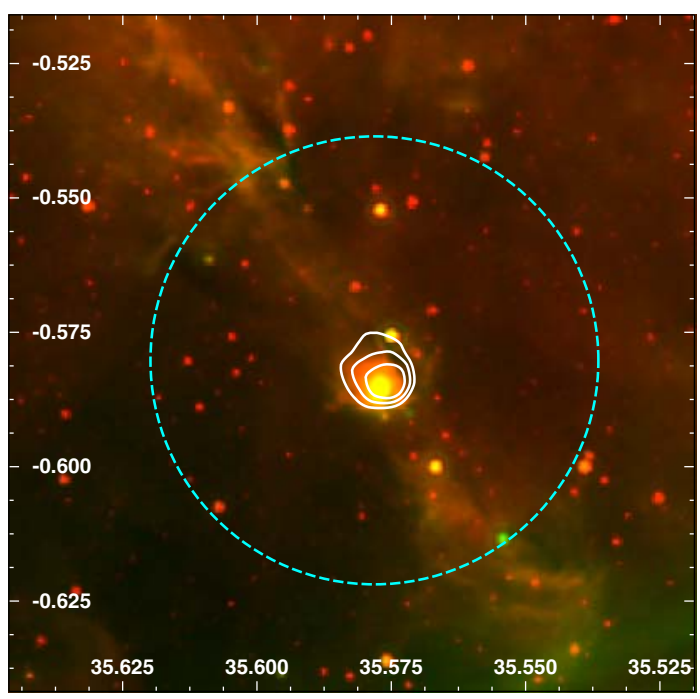

Fig. 3. Two-color image with the $8 \mu \mathrm{m}$ and $24 \mu \mathrm{m}$ emissions presented in red and green, respectively. The contours correspond to the ${ }^{12} \mathrm{CO} J=3-2$ emission integrated between 48 and $57 \mathrm{~km} \mathrm{~s}^{-1}$, at the levels of 22,26 , and $30 \mathrm{~K} \mathrm{~km} \mathrm{~s}^{-1}$. The rms noise is about $4 \mathrm{~K} \mathrm{~km} \mathrm{~s}^{-1}$. The circle represents the extension of the source HESS J1858+020.

Table 1. Parameters of the ${ }^{12} \mathrm{CO}$ and ${ }^{13} \mathrm{CO} J=3-2$ lines from the center of the region.

\begin{tabular}{lccc}
\hline \hline Emission & $\begin{array}{c}T_{\mathrm{mb}} \\
(\mathrm{K})\end{array}$ & $\begin{array}{c}V_{\mathrm{LSR}} \\
\left(\mathrm{km} \mathrm{s}^{-1}\right)\end{array}$ & $\begin{array}{c}\Delta v \\
\left(\mathrm{~km} \mathrm{~s}^{-1}\right)\end{array}$ \\
\hline${ }^{12} \mathrm{CO} J=3-2$ & $10.50 \pm 0.40$ & $53.15 \pm 0.22$ & $2.60 \pm 0.15$ \\
${ }^{13} \mathrm{CO} J=3-2$ & $5.20 \pm 0.50$ & $53.30 \pm 0.15$ & $2.00 \pm 0.10$ \\
\hline
\end{tabular}

the horizontal axis of each spectra is velocity and ranges from 30 to $80 \mathrm{~km} \mathrm{~s}^{-1}$, while the vertical axis is brightness temperature and goes from -1 to $7 \mathrm{~K}$. The ${ }^{12} \mathrm{CO} \mathrm{J}=3-2$ component at $\sim 64 \mathrm{~km} \mathrm{~s}^{-1}$ has no correspondence neither in the ${ }^{13} \mathrm{CO} J=3-2$ emission presented in this work, nor in the ${ }^{13} \mathrm{CO} J=1-0$ emission analyzed in Paron \& Giacani (2010). We suggest that this velocity component can be unrelated molecular gas seen along the line of sight. In what follows, we focus our analysis on the $\sim 53 \mathrm{~km} \mathrm{~s}^{-1}$ molecular component. Table 1 summarizes the derived parameters of the ${ }^{12} \mathrm{CO}$ and ${ }^{13} \mathrm{CO} J=3-2$ lines obtained from a Gaussian fitting. $T_{\mathrm{mb}}$ is the main beam peak brightness temperature, $V_{\mathrm{LSR}}$ is the central velocity referred to the local standard of rest and $\Delta v$ is the line width $(F W H M)$. The Gaussian fitting was performed on the averaged spectrum of each line, which was obtained from the pointings within the area mapped by the ${ }^{13} \mathrm{CO}$ emission, at the center of the region. The quoted uncertainties are formal $1 \sigma$ value for the model of the Gaussian shape.

An inspection of the ${ }^{12} \mathrm{CO} J=3-2$ spectra indicates that there are neither spectral wings nor intensity gradients along symmetric directions in the plane of the sky, which allows us to conclude that, at the present data resolution, there is no evidence of outflow activity neither in the plane of the sky nor along the line of sight. The detected molecular clump peaks approximately at the $(10,0)$ offset (see Fig. 2), corresponding to the sky positon $l=35.57, b=-0.58$. Figure 3 displays a two color image with the $8 \mu \mathrm{m}$ and $24 \mu \mathrm{m}$ emissions shown in red and green, respectively, with contours of the ${ }^{12} \mathrm{CO} J=3-2$ emission integrated between 48 and $57 \mathrm{~km} \mathrm{~s}^{-1}$. The circle represents the source HESS J1858+020. From this image, it can be appreciated that the molecular clump mapped in ${ }^{12} \mathrm{CO} J=3-2$ coincides with the condensation of PAHs seen at $8 \mu \mathrm{m}$. This image reveals that this clump also emits at $24 \mu \mathrm{m}$, indicating warm dust. This clump, lying exactly at the geometric center of the HESS source, suggests that its study may help us to elucidate the nature of the high energy emission. We note that we did not detect any emission from the $\mathrm{HCO}^{+} J=4-3$ and CS $J=7-6$ lines at sensitivity levels of about 0.13 and $0.2 \mathrm{~K}$, respectively, in the direction of this molecular concentration.

To estimate the physical parameters of the molecular clump, we assume LTE conditions and a beam filling factor of 1 , which may not be completely true but allows us to make a first approach to the problem. From the peak temperature ratio of the CO isotopes $\left({ }^{12} T_{\mathrm{mb}} /{ }^{13} T_{\mathrm{mb}}\right)$, it is possible to estimate the optical depths from (e.g. Curtis et al. 2010)

$\frac{{ }^{12} T_{\mathrm{mb}}}{{ }^{13} T_{\mathrm{mb}}}=\left(\frac{v_{12}}{v_{13}}\right)^{2} \frac{\left[{ }^{12} \mathrm{CO}\right]}{\left.{ }^{13} \mathrm{CO}\right]} \frac{1-\exp \left(-\tau_{12}\right)}{\tau_{12}}$,

where $v_{12}=345.796 \mathrm{GHz}$ and $v_{13}=330.558 \mathrm{GHz}$ are the transition frequencies of ${ }^{12} \mathrm{CO}$ and ${ }^{13} \mathrm{CO} J=3-2$ lines, respectively, $\tau_{12}$ is the optical depth of the ${ }^{12} \mathrm{CO}$ gas, and $\left[{ }^{12} \mathrm{CO}\right] /\left[{ }^{13} \mathrm{CO}\right]$ is the isotope abundance ratio. Assuming that $8 \mathrm{kpc}$ is the distance to the Galactic center and using $\left[{ }^{12} \mathrm{CO}\right] /\left[{ }^{13} \mathrm{CO}\right]=(6.21 \pm$ $1.00) D_{\mathrm{GC}}+(18.71 \pm 7.37)\left(\right.$ Milam et al. 2005) where $D_{\mathrm{GC}}=$ $6.73 \mathrm{kpc}$ is the distance between the source and the Galactic center, we obtain $\left[{ }^{12} \mathrm{CO}\right] /\left[{ }^{13} \mathrm{CO}\right]=56.7 \pm 13.5$. Thus, the ${ }^{12} \mathrm{CO} J=$ $3-2$ optical depth is $\tau_{12}=32 \pm 11$. Using the typical LTE equations and taking into account that the ${ }^{12} \mathrm{CO} J=3-2$ line is optically thick as shown above, from its emission we estimate an excitation temperature of $T_{\mathrm{ex}}=17 \pm 1 \mathrm{~K}$. Using this factor and the ${ }^{13} \mathrm{CO} J=3-2$ emission, we derive an optical depth for the ${ }^{13} \mathrm{CO}$ of $\tau^{13}=0.70 \pm 0.12$ and a ${ }^{13} \mathrm{CO}$ column density of $N\left({ }^{13} \mathrm{CO}\right)=(8.2 \pm 1.2) \times 10^{15} \mathrm{~cm}^{-2}$. Adopting the isotope abundance ratio $\left[{ }^{12} \mathrm{CO}\right] /\left[{ }^{13} \mathrm{CO}\right]$ used above and the relationship of $N\left(\mathrm{H}_{2}\right) / N\left({ }^{12} \mathrm{CO}\right) \sim 10^{4}$ (see Black \& Willner 1984, and reference therein), we obtain an $\mathrm{H}_{2}$ column density of $N\left(\mathrm{H}_{2}\right)=$ $(5.0 \pm 1.8) \times 10^{21} \mathrm{~cm}^{-2}$. Finally, assuming spherical geometry for the clump, which is compatible with what is seen in Fig. 3, with a radius of $\sim 30^{\prime \prime}(\sim 1.5 \mathrm{pc}$ at the distance of $10.5 \mathrm{kpc})$, we estimate a mass and a volume density of $(1.5 \pm 0.5) \times 10^{3} \times\left(\frac{d}{10.5 \mathrm{kpc}}\right)^{2} M_{\odot}$, and $(2.1 \pm 0.7) \times 10^{3} \times\left(\frac{10.5 \mathrm{kpc}}{d}\right)^{3} \mathrm{~cm}^{-3}$, respectively for this structure, where $d$ is the distance. The quoted errors, of the order of $30 \%$, do not include the error in the distance, which is a major unknown and depends on Galaxy models. We thus present the estimated values as a function of the distance.

Using the ${ }^{13} \mathrm{CO}$ line width of $\Delta v=2 \mathrm{~km} \mathrm{~s}^{-1}$ and a radius of $R=1.5 \mathrm{pc}$, we also calculate the virial mass from $M_{\text {vir }}=B \times R \times \Delta v^{2}$, where $B$ is a constant that depends on the density profile. If one assumes a uniform density profile, that is $\rho(r)=$ const., $B=210$, while if a density profile $\rho(r) \propto 1 / r$ is assumed, $B=190$ (MacLaren et al. 1988). Both cases produce the same virial mass within the errors, $M_{\mathrm{vir}}=$ $(1.1 \pm 0.1) \times 10^{3} \times \frac{d}{10.5 \mathrm{kpc}} M_{\odot}$. The ratio of the virial and the LTE mass is $M_{\mathrm{vir}} / M_{\mathrm{LTE}}=(0.8 \pm 0.3) \times \frac{10.5 \mathrm{kpc}}{d}$. Kawamura et al. (1998) performed a large-scale survey of molecular clouds towards the Gemini and Auriga regions and shows that starforming ${ }^{13} \mathrm{CO}$ clouds have low $M_{\mathrm{vir}} / M_{\mathrm{LTE}}$, while all the clouds with high $M_{\mathrm{vir}} / M_{\mathrm{LTE}}$ have no sign of star formation. On the other hand, several molecular cores studied in the active star forming complex in Taurus have on average, $M_{\text {vir }} / M_{\text {LTE }} \sim 0.6$ (Onishi et al. 1996), which is quite similar to the value of 0.8 derived here.

Another way to estimate the volume density of the molecular feature is by investigating of the dust content. Figure 4 displays 
Table 2. Near- and mid-IR fluxes of IRS1 and IRS2.

\begin{tabular}{lcccccccc}
\hline \hline Source & $\begin{array}{c}J \\
(\mathrm{mag})\end{array}$ & $\begin{array}{c}H \\
(\mathrm{mag})\end{array}$ & $\begin{array}{c}K_{\mathrm{S}} \\
(\mathrm{mag})\end{array}$ & $\begin{array}{c}3.6 \mu \mathrm{m} \\
(\mathrm{mag})\end{array}$ & $\begin{array}{c}4.5 \mu \mathrm{m} \\
(\mathrm{mag})\end{array}$ & $\begin{array}{c}5.8 \mu \mathrm{m} \\
(\mathrm{mag})\end{array}$ & $\begin{array}{c}8.0 \mu \mathrm{m} \\
(\mathrm{mag})\end{array}$ & $\begin{array}{c}24 \mu \mathrm{m} \\
(\mathrm{Jy})\end{array}$ \\
\hline IRS1 & - & - & 13.462 & 10.253 & 9.225 & 8.301 & 7.533 & 0.032 \\
IRS2 & 15.318 & 13.344 & 12.342 & 10.731 & 10.137 & 9.625 & 9.053 & 0.150 \\
\hline
\end{tabular}

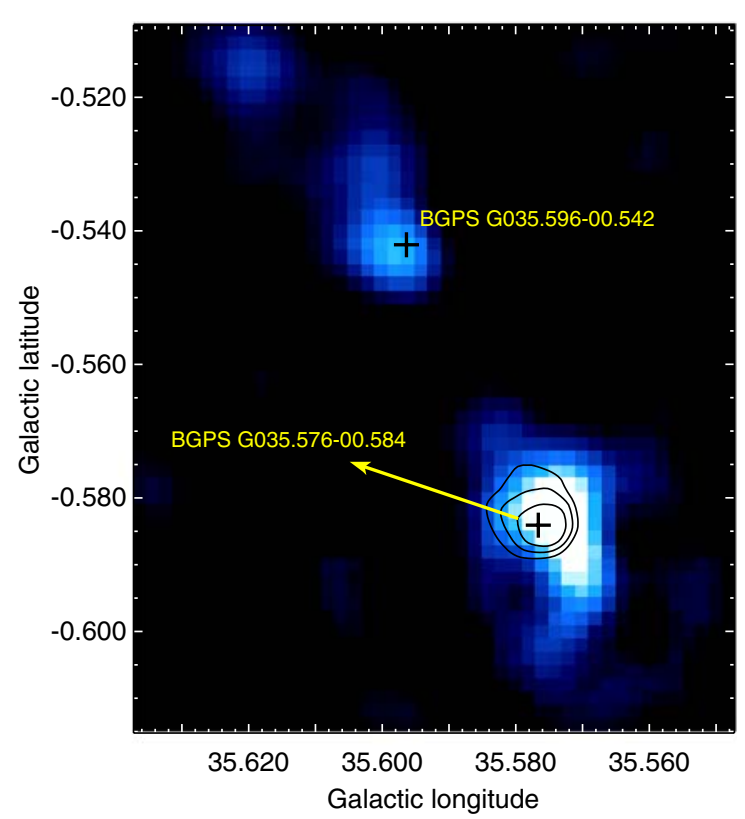

Fig. 4. BGPS continuum emission at $1.1 \mathrm{~mm}$ with the ${ }^{12} \mathrm{CO} J=3-2$ contours presented in Fig. 3. The crosses indicate the position of two sources catalogued in the BGPS catalog.

the smoothed $1.1 \mathrm{~mm}$ continuum emission obtained from the BGPS (Aguirre et al. 2011) with the ${ }^{12} \mathrm{CO} J=3-2$ contours presented in Fig. 3. The crosses indicate the position of two sources from the BGPS catalog (Rosolowsky et al. 2010), showing that the analyzed molecular clump coincides with the source BGPS G035.578-00.584.

According to the BGPS catalog, the source BGPS G035.57800.584 has an integrated flux density at $1.1 \mathrm{~mm}$ of $S_{v}=0.37 \pm$ $0.10 \mathrm{Jy}$ and an elliptical shape with a major and minor axis of $16 . ' 9$ and 13.' 6 , respectively. The total mass of gas and dust in a core is proportional to the total flux density $S_{v}$, assuming that the dust emission at $1.1 \mathrm{~mm}$ is optically thin and both the dust temperature and opacity are independent of position within the core (Enoch et al. 2006). We can then calculate the core mass from the BGPS G035.578-00.584 flux density using

$$
M=\frac{d^{2} S_{v}}{B_{v}\left(T_{\mathrm{d}}\right) \kappa_{v}}
$$

where $\kappa_{1.1 \mathrm{~mm}}=0.0114 \mathrm{~cm}^{2} \mathrm{~g}^{-1}$ is the dust opacity estimated on the basis of the canonical gas-to-dust mass ratio of 100 (Enoch et al. 2006), $d$ the distance, $B_{v}$ the Planck function, and $T_{\mathrm{d}}$ the dust temperature. Although the millimeter emission arises only from the dust, it is possible to infer the total mass of gas and dust because, as mentioned above, the dust opacity $\kappa_{1.1} \mathrm{~mm}$ contains the gas-to-dust mass ratio (see Enoch et al. 2006). Following Rosolowsky et al. (2010), the above equation can be written as

$$
M=13.1 M_{\odot}\left(\frac{d}{1 \mathrm{kpc}}\right)^{2}\left(\frac{S_{v}}{1 \mathrm{Jy}}\right)\left[\frac{\exp \left(13 / T_{\mathrm{d}}\right)-1}{\exp (13 / 20)-1}\right] .
$$

Assuming a typical dust temperature of $T_{\mathrm{d}}=20 \mathrm{~K}$ and a distance of $10.5 \mathrm{kpc}$, we obtain a total mass for the core BGPS G035.57800.584 of $M=(535 \pm 15) \times\left(\frac{d}{10.5 \mathrm{kpc}}\right)^{2} M_{\odot}$. Finally, using this mass and assuming an angular radius of $14^{\prime \prime}(R \sim 0.7 \mathrm{pc})$, from $n_{\mathrm{H}}=3 M /\left(4 \pi R^{3} \mu m_{\mathrm{H}}\right)$, where $m_{\mathrm{H}}$ is the hydrogen atom mass and $\mu=2.37$ the mean particle mass, we obtain a particle density of $n_{\mathrm{H}}=(6.6 \pm 0.2) \times 10^{3} \times\left(\frac{10.5 \mathrm{kpc}}{d}\right)^{3} \mathrm{~cm}^{-3}$. The adopted angular radius is based on the angular size of this object reported in the catalog. As can be appreciated, the minor axis size is smaller than the rms size of the BGPS beam, thus it is not possible to calculate the deconvolved angular radius following Rosolowsky et al. (2010).

In summary, from two different methods we obtain a density of a few $10^{3} \mathrm{~cm}^{-3}$ for the studied clump. Taking into account the lack of emission of the CS $J=7-6$ and $\mathrm{HCO}^{+} J=4-3$ lines, tracers of higher densities, we conclude that $10^{3}-10^{4} \mathrm{~cm}^{-3}$ is a plausible range for the density in the clump.

\subsection{Young stellar objects in the molecular clump}

Paron \& Giacani (2010) conducted a search for young stellar objects (YSOs) probably embedded in the molecular cloud mapped in the ${ }^{13} \mathrm{CO} J=1-0$ line. Using the color criteria of Allen et al. (2004) for GLIMPSE sources, the authors found six YSO candidate sources. In this work, we search for YSOs probably embedded in the discovered ${ }^{12} \mathrm{CO} J=3-2$ clump using criteria based on the intrinsic reddening of the sources and studying the physical parameters extracted from the YSOs spectral energy distributions (SEDs). These criteria assume that YSOs always display an intrinsic infrared excess that cannot be attributed to scattering and/or absorption of the ISM along the line of sight. We therefore used the GLIMPSE Point-Source Catalog to search for this kind of sources within the molecular clump. Robitaille et al. (2008) defined a color criterion to identify intrinsically red sources using data from the Spitzer-IRAC bands. Intrinsically red sources satisfy the condition $m_{4.5}-m_{8.0} \geq 1$, where $m_{4.5}$ and $m_{8.0}$ are the magnitudes in the 4.5 and $8.0 \mu \mathrm{m}$ bands, respectively. To consider the errors in the magnitudes, we use the following color criterion to select intrinsically red sources $m_{4.5}-m_{8.0}+\varepsilon \geq 1$, where $\varepsilon=\sqrt{\left(\Delta_{4.5}\right)^{2}+\left(\Delta_{8.0}\right)^{2}}$ and $\Delta_{4.5}$ and $\Delta_{8.0}$ are the errors in the 4.5 and $8.0 \mu \mathrm{m}$ bands, respectively. By inspecting a circular region of about $30^{\prime \prime}$ in radius centered at $l=35.578, b=-0.582$, we find 27 GLIMPSE sources, and by applying the abovementioned color criterion, we find only two intrinsically red sources that appear to be related to the molecular clump, called SSTGLMC G035.5768-00.5862 and SSTGLMC G035.5765-00.5909, hereafter IRS1 and IRS2, respectively. These sources were classified as class I and II, respectively in Paron \& Giacani (2010) following the Allen et al. (2004) classification. In view of our more complete study, we now suggest that IRS1 is very likely to be embedded in the analyzed molecular clump, while for IRS2, lying on the border of the observed region, the connection with the studied molecular feature is less compelling (see Fig. 5 left).

In Table 2, we present the catalogued near- and mid-IR fluxes of these sources extracted from the 2MASS and GLIMPSE point 

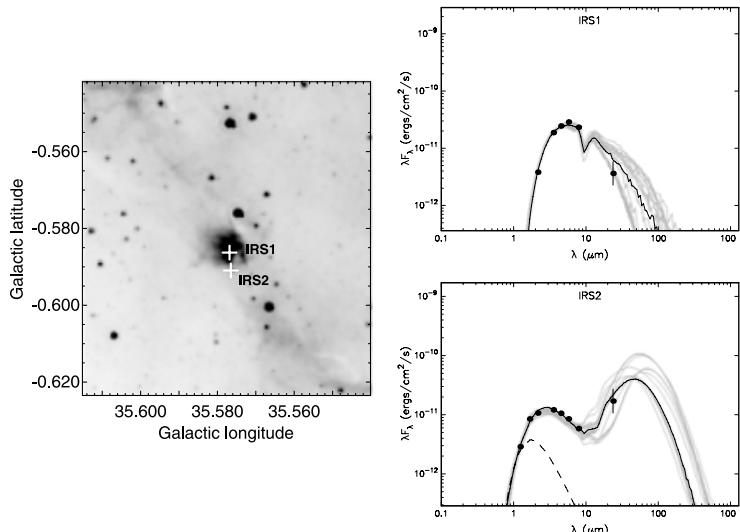

Fig. 5. Left: location of sources IRS1 and IRS2. Right: SED of these sources performed with the tool developed by Robitaille et al. (2006, 2007). Black line shows the best fit, and the gray lines show subsequent good fits. The dashed line shows the stellar photosphere corresponding to the central source of the best fit model, as it would look in the absence of circumstellar dust. The points are the input fluxes.

Table 3. Parameters derived from the SED fitting of sources IRS1 and IRS2.

\begin{tabular}{lcccccc}
\hline \hline Source & $\chi_{\text {YSO }}^{2}$ & $\chi_{\star}^{2}$ & $\begin{array}{c}M_{\star} \\
\left(M_{\odot}\right)\end{array}$ & $\begin{array}{c}M_{\text {disk }} \\
\left(M_{\odot}\right)\end{array}$ & $\begin{array}{c}M_{\text {env }} \\
\left(M_{\odot}\right)\end{array}$ & $\begin{array}{c}\dot{M}_{\text {env }} \\
\left(M_{\odot} / \mathrm{yr}\right)\end{array}$ \\
\hline IRS1 & 4.1 & 412 & 9.6 & $3.2 \times 10^{-2}$ & $3.6 \times 10^{-6}$ & 0 \\
IRS2 & 4.6 & 166 & 6.4 & $3.3 \times 10^{-2}$ & 1.7 & $5 \times 10^{-6}$ \\
\hline
\end{tabular}

source catalogs. The fluxes at $24 \mu \mathrm{m}$ were obtained from the MIPS image. These fluxes were used to calculate the SED of IRS1 and IRS2 using the tool developed by Robitaille et al. (2006, 2007), which is available online ${ }^{1}$. To compile the SED, we assume an interstellar absorption of between 10 and 30 mag. The lower value is compatible with the rough assumption of $1 \mathrm{mag}$ per kpc that is commonly used. The upper value agrees with the visual absorption obtained from $A_{\mathrm{v}}=5 \times 10^{-22} N(\mathrm{H})$ (Bohlin et al. 1978) where $N(\mathrm{H})=N(\mathrm{HI})+2 N\left(\mathrm{H}_{2}\right)$ is the lineof-sight hydrogen column density towards this region, which we estimate to be about $5.8 \times 10^{22} \mathrm{~cm}^{-2}$. This value was obtained from the HI column density derived from the VLA Galactic Plane Survey (VGPS) HI data (Stil et al. 2006) and from the $\mathrm{H}_{2}$ column density derived above. In Table 3, we report the main results of the SED fit output for IRS1 and IRS2. In Cols. 2 and 3, we report the $\chi^{2}$ of the YSO and stellar photosphere best-fit model, respectively. The remaining columns report the physical parameters of the source for the best-fit model: central source mass, disk mass, envelope mass, and envelope accretion rate, respectively. Figure 5 right shows the SED of these sources.

To relate the SED to the evolutionary stage of the YSO, Robitaille et al. (2006) defined three different stages based on the values of the central source mass $M_{\star}$, the disk mass $M_{\text {disk }}$, and the envelope accretion rate $\dot{M}_{\text {env }}$ of the YSO. Stage I YSOs are those that have $\dot{M}_{\text {env }} / M_{\star}>10^{-6} \mathrm{yr}^{-1}$, i.e., protostars with large accretion envelopes; stage II are those with $M_{\text {disk }} / M_{\star}>10^{-6}$ and $\dot{M}_{\text {env }} / M_{\star}<10^{-6} \mathrm{yr}^{-1}$, i.e., young objects with prominent disks; and stage III are those with $M_{\text {disk }} / M_{\star}<10^{-6}$ and $\dot{M}_{\text {env }} / M_{\star}<10^{-6} \mathrm{yr}^{-1}$, i.e. evolved sources where the flux is dominated by the central source. According to this classification, we conclude that IRS1 and IRS2 are stage II sources. However, IRS2 must be younger than IRS1 because, as can be appreciated

\footnotetext{
${ }^{1}$ http://caravan.astro.wisc.edu/protostars/
}

in Table 3, it has a massive envelope that must still be accreting mass. The evolutionary stage of IRS1 derived from its SED and the lack of evidence of molecular outflows in the clump where it is embedded, suggest that IRS1 is an evolved YSO probably in the last stages of formation. Moreover, the presence of a condensation of PAH around this source suggests that IRS1 could be a high-mass protostellar object (HMPO) that has not yet reached the ultracompact HII region stage.

\subsection{The scenario}

On the basis of the results presented above, we discuss the possible origin of the very high-energy emission.

As mentioned in Sect. 3, we propose that the SNR G35.6-0.4 partially overlaps an extended HII region, whose eastern border is delineated by PAHs revealed by the $8 \mu \mathrm{m}$ emission. A molecular cloud composed of at least two clumps lies over this border, and one of them is located at the center of HESS J1858+02. We have shown that there is at least one YSO embedded in this clump (that we called IRS1), which can, in principle, create a population of relativistic particles inside the host molecular cloud via a thermal jet. These particles, in a high density ambient environment matter can produce $\gamma$-ray emission by means of inverse Compton and relativistic bremsstrahlung losses (Araudo et al. 2007). However, for IRS1, at the present data resolution, no evidence of molecular outflows has been found in either the plane of the sky or along the line of sight, therefore weakening the probability of a physical link between IRS1 and HESS J1858+020. Since we have demonstrated that a YSO in the molecular clump is unlikely to play a decisive role in producing the observed $\gamma$-rays, and because of the lack of any other candidate in the region at any distance, we conclude that the only possible Galactic counterpart to the HESS source is the SNR G35.6-0.4 with its molecular enviroment. In this case, the supernova shock is a source of accelerated cosmic rays and the dense molecular clump provides the nuclei responsible for the production of neutral pions (by means of inelastic pp collisions), which will decay yielding the observed $\gamma$-rays.

\section{Summary}

Using molecular observations obtained with the Atacama Submillimeter Telescope Experiment (ASTE) and IR and submillimeter continuum archival data, we have studied a molecular clump associated with the IR source IRAS $18558+0201$ that lies at the center of the very-high energy source HESS J1858+020. Our main results can be summarized as follows:

(a) From the ${ }^{12} \mathrm{CO}$ and ${ }^{13} \mathrm{CO} J=3-2$ lines and the $1.1 \mathrm{~mm}$ continuum emission for this clump we have measured a density between $10^{3}$ and $10^{4} \mathrm{~cm}^{-3}$. This clump is part of a larger molecular cloud that is being disturbed by the SNR G35.60.4 and a nearby extended HII region.

(b) From the analysis of the mid-IR data and a photometric study, we have discovered a YSO very likely embedded in the aforementioned molecular clump. Analyzing its spectral energy distribution, we suggest that this source could be a high-mass protostellar object that has not yet reached the ultracompact HII region stage.

(c) We did not find any evidence of molecular outflows from the discovered YSO that would reveal the presence of a thermal jet capable by itself of generating the very-high energy emission. 
(d) We conclude that a clumpy molecular cloud, similar to the one investigated in this work, is the most plausible explanation of the very-high energy emission. The molecular gas may be acting as a target for the cosmic rays accelerated by the shock front of the SNR G35.6-0.4 generating the $\gamma$-ray emission by means of hadronic processes.

Acknowledgements. S.P., E.G. and G.D. are members of the Carrera del Investigador Cientifico of CONICET, Argentina. This work was partially supported by Argentina grants awarded by Universidad de Buenos Aires, CONICET and ANPCYT. M.R. wishes to acknowledge support from FONDECYT (CHILE) grant No. 108033. She is supported by the Chilean Center for Astrophysics FONDAP No. 15010003. S.P. and M.R. are grateful to Dr. Shinya Komugi for the support received during the observations. We wish to thank the anonymous referee whose comments and suggestions have helped to improve the paper.

\section{References}

Aguirre, J. E., Ginsburg, A. G., Dunham, M. K., et al. 2011, ApJS, 192, 4 Aharonian, F., Akhperjanian, A. G., Barres de Almeida, U., et al. 2008, A\&A, 477,353

Allen, L. E., Calvet, N., D'Alessio, P., et al. 2004, ApJS, 154, 363
Araudo, A. T., Romero, G. E., Bosch-Ramon, V., \& Paredes, J. M. 2007, A\&A, 476, 1289

Black, J. H., \& Willner, S. P. 1984, ApJ, 279, 673

Bohlin, R. C., Savage, B. D., \& Drake, J. F. 1978, ApJ, 224, 132

Curtis, E. I., Richer, J. S., Swift, J. J., \& Williams, J. P. 2010, MNRAS, 408 1516

Enoch, M. L., Young, K. E., Glenn, J., et al. 2006, ApJ, 638, 293

Ezawa, H., Kawabe, R., Kohno, K., \& Yamamoto, S. 2004, in SPIE Conf. 5489, ed. J. M. Oschmann, Jr., 763

Green, D. A. 2009, MNRAS, 399, 177

Helou, G., \& Walker, D. W. 1988, Infrared astronomical satellite (IRAS) catalogs and atlases, The small scale structure catalog, 7

Kawamura, A., Onishi, T., Yonekura, Y., et al. 1998, ApJS, 117, 387

MacLaren, I., Richardson, K. M., \& Wolfendale, A. W. 1988, ApJ, 333, 821

Milam, S. N., Savage, C., Brewster, M. A., Ziurys, L. M., \& Wyckoff, S. 2005, ApJ, 634, 1126

Onishi, T., Mizuno, A., Kawamura, A., Ogawa, H., \& Fukui, Y. 1996, ApJ, 465, 815

Paron, S., \& Giacani, E. 2010, A\&A, 509, L4

Robitaille, T. P., Whitney, B. A., Indebetouw, R., Wood, K., \& Denzmore, P. 2006, ApJS, 167, 256

Robitaille, T. P., Whitney, B. A., Indebetouw, R., \& Wood, K. 2007, ApJS, 169, 328

Robitaille, T. P., Meade, M. R., Babler, B. L., et al. 2008, AJ, 136, 2413

Rosolowsky, E., Dunham, M. K., Ginsburg, A., et al. 2010, ApJS, 188, 123

Stil, J. M., Taylor, A. R., Dickey, J. M., et al. 2006, AJ, 132, 1158 